\title{
Murine colon proteome and characterization of the protein pathways
}

Sameh Magdeldin ${ }^{1,2^{*}}$, Yutaka Yoshida ${ }^{1}$, Huiping Li ${ }^{1,3}$, Yoshitaka Maeda ${ }^{4}$, Munesuke Yokoyama ${ }^{4}$, Shymaa Enany ${ }^{1,5}$, Ying Zhang ${ }^{1}$, Bo Xu ${ }^{1}$, Hidehiko Fujinaka', Eishin Yaoita ${ }^{1}$, Sei Sasaki ${ }^{6}$ and Tadashi Yamamoto ${ }^{1}$

\author{
* Correspondence: samehmagd@ \\ yahoo.com \\ ${ }^{1}$ Department of Structural \\ Pathology, Institute of Nephrology, \\ Graduate School of Medical and \\ Dental Sciences, Niigata University, \\ Niigata, Japan \\ ${ }^{2}$ Department of Physiology, Faculty \\ of Veterinary Medicine, Suez Canal \\ University, Suez Canal, Egypt \\ Full list of author information is \\ available at the end of the article
}

\begin{abstract}
Background: Most of the current proteomic researches focus on proteome alteration due to pathological disorders (i.e.: colorectal cancer) rather than normal healthy state when mentioning colon. As a result, there are lacks of information regarding normal whole tissue- colon proteome.

Results: We report here a detailed murine (mouse) whole tissue- colon protein reference dataset composed of 1237 confident protein $(F D R<2)$ with comprehensive insight on its peptide properties, cellular and subcellular localization, functional network GO annotation analysis, and its relative abundances. The presented dataset includes wide spectra of $\mathrm{p} /$ and Mw ranged from 3-12 and 4-600 KDa, respectively. Gravy index scoring predicted $19.5 \%$ membranous and $80.5 \%$ globularly located proteins. GO hierarchies and functional network analysis illustrated proteins function together with their relevance and implication of several candidates in malignancy such as Mitogen- activated protein kinase (Mapk8, 9) in colorectal cancer, Fibroblast growth factor receptor (Fgfr 2), Glutathione S-transferase (Gstp1) in prostate cancer, and Cell division control protein (Cdc42), Ras-related protein (Rac1,2) in pancreatic cancer. Protein abundances calculated with 3 different algorithms (NSAF, PAF and emPAl) provide a relative quantification under normal condition as guidance.

Conclusions: This highly confidence colon proteome catalogue will not only serve as a useful reference for further experiments characterizing differentially expressed proteins induced from diseased conditions, but also will aid in better understanding the ontology and functional absorptive mechanism of the colon as well.
\end{abstract}

Keywords: Colon, Proteome, Mass spectrometry, HPLC

\section{Background}

The essential role of colon in normal physiology includes absorption of vitamins, salts, nutrients and water which is summarized as the final stage digestive process [1,2]. Together with its ability to process indigestible fibers (in certain species) [3], it hosts a wide range of useful microbiota which live in a symbiotic relationship providing a proper absorption and wastes elimination [4]. Recruitment of proteomics in colon studies has been an interest for many researchers especially gastroenterologists [5]. However, overwhelming majorities of these reports were focusing on proteome of disordered colon tissues rather than its normal state. Owing to the Pubmed-Medline search result with the keywords "Colon" and "Proteomics" on June 2012, 300 literatures

(c) 2012 Magdeldin et al.; licensee BioMed Central Ltd. This is an Open Access article distributed under the terms of the Creative Commons Attribution License (http://creativecommons.org/licenses/by/2.0), which permits unrestricted use, distribution, and reproduction in any medium, provided the original work is properly cited. 
investigated diseased-state colon proteome (mainly colorectal cancer), while less than 5 partially reported healthy one. To this end, the availability of comprehensive whole tissue- colon proteome remains a critical demand. In a former report by Gourley et al..., the authors compared colonic gastrointestinal mucosa by 2 different approaches; 2D and 2DLC. Integration of both analyses resulted in identification of 568 proteins [5]. Another 2DE study examining colonic crypts identified approximately 800 proteins [6], while other comparative experiments compared healthy intestinal scraping versus cell lines (Caco-2) [7], aged intestinal epithelia [8] or colorectal cancer [9-11]. Given the necessity of disclosing colon proteome in its normal state to overcome currently insufficient global understanding of physiological and pathophysiological colonic tissues profiles, our impetus was to provide a comprehensive whole tissue colon proteome as a mainstay for further colon research analysis.

\section{Materials and methods}

Animals

Three healthy normal C57BL/6 J male mice (8 weeks old) bred at the animal Care Research Center (Niigata University, Japan) was used in the current study. Mice were housed in individual cages in sterile environment with 12 hour light cycle and ad lib access to standard chow and filtered water. Experimental animal were treated in accordance with ethics of animal center committee at school of medical and dental sciences, Niigata University [approval number 2009-32].

\section{Tissue processing and protein extraction}

Mice were sacrificed by decapitation and colon; $3 \mathrm{~cm}$ above the rectum were longitudinally cut, and rinsed in ice-cold PBS buffer. Tissues were sliced into small pieces prior to homogenization. For colonic protein extraction, $100 \mathrm{mg}$ of wet tissue were homogenized in home- made lysis solution consists of (9.8 M urea, 2\% nonidet, $0.2 \%$ Ampholite; $\mathrm{pH}$ 3-10, $12 \mu \mathrm{l} / \mathrm{ml}$ Destreak buffer, $0.5 \mu \mathrm{g} / \mathrm{ml}$ E-64, $0.5 \mathrm{mM}$ PMSF, $40 \mu \mathrm{g} /$ $\mathrm{ml}$ TLCK, $1.0 \mu \mathrm{g} / \mu \mathrm{l}$ chymostatin, $0.5 \mathrm{mM}$ EDTA, $0.01 \%$ bromophenol blue, and $2 \mu \mathrm{g} / \mu \mathrm{l}$ aprotonin). Samples were homogenized using Polytron PT1200 homogenizer (Kinematica AG, Switzerland) 5-10 bursts with $45 \mathrm{~s}$ interval in ice. Homogenized samples were kept in $37^{\circ} \mathrm{C}$ for $1 \mathrm{~h}$ with occasional vortex, centrifuged at 12,000 rpm for $20 \mathrm{~m}$. Protein assay for the extracts was carried out using Ramagli's modified method of Bradford (Bio-Rad, Japan) with bovine serum albumin as a standard [12].

\section{Protein fractionation, tryptic digestion}

Samples were acetone precipitated and reconstituted in SDS sample buffer with $2 \beta$ mercaptoethanol (final dilution 4\%) [13]. Ten $\mu \mathrm{g}$ of colon protein extract from each sample were run on 12.5\% SDS-PAGE. Gel was stained with Coomassie Brilliant Blue stain (CBB R-250, Wako, Japan). Each lane was sliced into 14 consecutive slices $(2 \mathrm{~cm} /$ slice). Samples were reduced with $10 \mathrm{mM}$ dithiothreitol (DTT), alkylated with $55 \mathrm{mM}$ iodoacetamide (IAA), and digested with $6 \mathrm{ng} / \mu \mathrm{l}$ of trypsin overnight [14]. Peptide was extracted with $0.3 \%$ formic acid and $5 \mu \mathrm{l}(0.25 \mu \mathrm{g}$ digested protein) from each sample was loaded onto nano-LC-ESI-IT-TOF-MS/MS (Hitachi NanoFrontier LD., Tokyo, Japan). 


\section{Reversed- phase capillary Lc-Ms/Ms analysis}

Digested peptides were purified and concentrated on a trap column; monolith trap C18-50-150 (Merck, Darmstadt, Germany). Peptides were separated using the C18 separation column; monocap for Fast-Flow, $0.05 \times 150 \mathrm{~mm}$. The injected peptides were eluted with $7.5-70 \%$ gradient with solvent $\mathrm{B}\left(\mathrm{H}_{2} \mathrm{O} / \mathrm{ACN}=98 / 2\right.$ in $\left.0.1 \% \mathrm{HCOOH}\right)$ for 120 minutes at $200 \mathrm{nl} /$ minute. Nano-LC-ESI-IT-TOF-MS/MS was performed on the top of two ions in each MS scan. Dynamic exclusion and repeat settings ensured each ion was selected only once and excluded in the subsequent parent ion selection. Precursor ions were selected using the following MS to MS/MS switch criteria: ion range $\mathrm{m} / \mathrm{z}$ 100-1800, charge state 2-5, and former target ion were excluded for $20 \mathrm{~ms}$. Collision ion dissociation (CID) was performed using nitrogen as collision gas. Data were merged using Mascot daemon (V 2.0) [15].

\section{Ms/Ms data processing and protein identification}

Peak lists were generated using NanoFrontier LD data processing software (V 1.0). Product ion data were searched against Mouse International protein index (IPI_mouse; version 3.71, 169347 entries) using a locally stored copy of the Mascot search engine (version 2.2.1, Matrix Science, London, UK) [15]. The following parameters were used for database search: MudPIT scoring, precursor mass tolerance $0.3 \mathrm{Da}$, product ion mass tolerance $0.3 \mathrm{Da}, 2$ missed cleavages allowed, fully tryptic peptides only, fixed modification of Carbamoidomethyl $(\mathrm{C})$, variable modifications of glutamine (Gln) to pyroglutamate (pyro-Glu) (N-term Q); glutamate (Glu) to pyroglutamate (pyro-Glu) (N-term E), Oxidation of histidine and tryptophan (HW); Oxidation of methionine (M), mass values of monoisotopic and peptide charge state of $2+$ and $3+$. Protein was accepted if at least 2 peptides passed identity and homology threshold of Mascot (MOWSE) algorithm [16]considering that if multiple spectra were identified to match precisely the same sequence and charge state of a given peptide, only the spectrum with highest score was retained. The false discovery rate (FDR) against reversed decoy database was below $2 \%$.

\section{Relative protein abundance and gene ontology (GO) annotation}

To estimate protein contents in the analyzed samples, normalized spectral abundance factor (NSAF) for each protein was calculated [17], in which the total number of tandem mass spectra $(\mathrm{SpC})$ matching peptides of a given protein was divided by its protein length $(\mathrm{L})$, then divided by the sum of $(\mathrm{SpC} / \mathrm{L})$ for all uniquely identified proteins in each dataset. Protein abundance factor (PAF) was calculated for each protein where PAF of a given protein is expressed as the total number of non redundant spectra normalized to the molecular weight (KDa) of the cognate protein $\left(10^{4}\right)$ [18]. Moreover, protein weight\% was estimated based on algorithms of exponentially modified protein abundance factor (emPAI) [19]. These parameters were used to rank proteins according to their relative abundance. In addition, enrichment, depletion analysis and functional annotation network for GO terms were visualized and statistically evaluated using BiNGO (v2.3) [20] and ClueGO (v1.1) plug-ins [21] integrated in Cytoscape (v 2.6.3) [22]. To access over- and under- represented GO hierarchies, both plug-ins were setup to two sided hypergeometric statistical testing with significance level $(\mathrm{P}<0.05)$. False 
discovery rate (FDR) correction was calculated using Benjamini and Hochberg multiple testing correction [23]. A customized and updated GO slim file (OBO v1.2; 32150 term) and gene annotation file were downloaded from GO consortium [24] and Kyoto encyclopedia of genes and genomes (KEGG) pathway databases [25], respectively and used in the current analysis. For secondary structure prediction of membrane and globular proteins, Gravy index (average hydrophobicity or hydrophilicity scores) were measured using Kyte-Doolittle and Hopp Woods formula [26].

\section{Results and discussion}

\section{Reproducibility}

At first, we examined the reproducibility of our experimental procedures and analysis. Colon tissues were carefully isolated from 3 normal healthy wild type mice. Protein was extracted, run on SDS-PAGE, stained and sliced as shown in Figure 1. Tryptic peptide mixture was eluted, pre-fractionated on $\mathrm{C} 18$ separation column and analyzed by NanoLC-ESI-IT-TOF-MS/MS. Reproducibility of mass analysis was confirmed by matching total ion chromatography (TIC) and peak retention time together with regular monitoring shifts between theoretical and calculated molecular weight precursor ions (Additional file 1). Moreover, stability of the mass spectrometer was confirmed by performing duplicate check analysis of the same sample which yielded $80-87 \%$ similarity of identified proteins (data are not shown). A total of 1237 high confidence proteins with 2 or more peptide matches and FDR $<2$ were identified after merging three data sets and removal of redundancy (based on the IPI accession number). Identified protein, including IPI accession number, protein name, peptide matches, theoretical and calculated MW and PI are listed in Additional file 2.

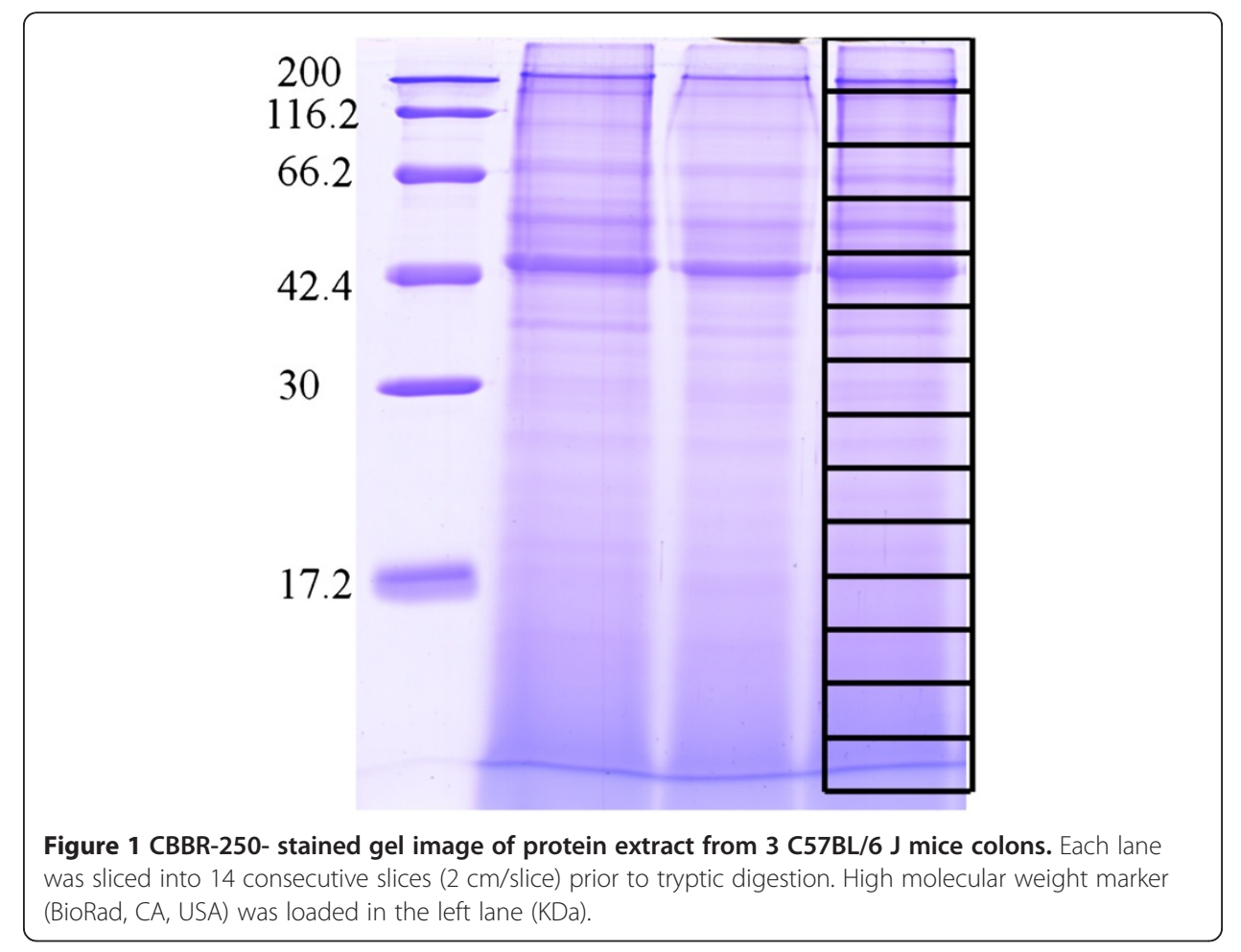




\section{Mouse colon proteome}

Using protein data sets generated from 42 slice analysis, we created a proteome catalogue of mouse colon by merging and refining outputs from redundancy and low confidence protein candidates with 1 peptide match. Protein candidate was accepted and considered a confident hit if at least 2 corresponding peptides passed identity and homology threshold of Mascot (MOWSE) algorithm [16]. Over $48.000 \mathrm{Ms} / \mathrm{Ms}$ spectra corresponding to 1237 protein candidate were detected and used to configure murine colon proteome (Additional file 2). Ms/Ms spectra of annotated peptides are freely accessible through proteomic data repository, PRIDE, the PRoteomic IDEntification database; http://www.ebi.ac.uk/pride/ under accession number [16857]. Constructed dataset was used for further characterization of mouse colon proteins.

\section{Characteristics of murine colon proteins}

As illustrated in Figures 2 and 3, we had characterized the identified proteins based on their $\mathrm{p} I, \mathrm{Mw}$ and hydrophobicity. While $\sim 68 \%(\mathrm{n}=843)$ of proteins were fallen in $\mathrm{p} I$ ranged from 5-9 and theoretical Mw 10 to $100 \mathrm{KDa}$, a number of proteins with extreme $\mathrm{p} I$ or $\mathrm{Mw}$ exists in our database; for instance, $\sim 12.5 \%$ of identified proteins $(\mathrm{n}=155)$ were reported with acidic $\mathrm{p} I$ ranged from 3-5 [Anp32a; $\mathrm{p} I$ 3.99]. Conversely, basic $\mathrm{p} I$ proteins ranged from $9-12$ were representing $~ 19.5 \%(\mathrm{n}=239)$ such as Sfrs7 ( $\mathrm{p} I$ 11.83). In addition, we succeeded to resolve proteins with wide $\mathrm{Mw}$ ranged from 4 to $600 \mathrm{KDa}$, for Hspb1 and Ahnak, respectively. These eccentric proteins would rarely be resolved by $2 \mathrm{DE}$. In viewing cellular localization of constructed colon proteome, we further categorized protein candidates into globular or membranous proteins based on amino acids hydrophobicity calculated by Kyte-Doolittle and Hopp Woods formula.

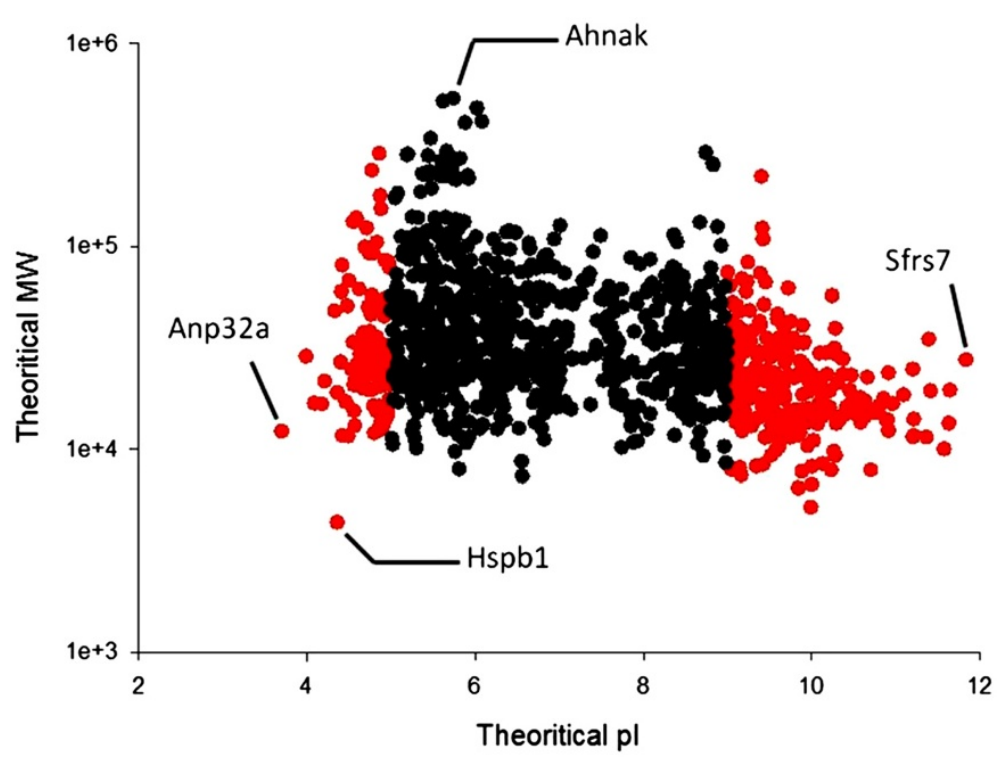

Figure 2 Theoretical pl and MW (Da) of identified proteins $(n=1237)$. Black spots represent candidates with p/ ranged 5 to 9. Red spots represent eccentric proteins with acidic p/ (3-5) or basic p/ (9-12). Hspb1; Truncated heat shock protein $1[\mathrm{MW}<4 \mathrm{KDa}$. Ahnak; neuroblast differentiation- associated protein [MW 600 $\mathrm{KDa}$ ]. Sfrs 7 ; isoforms 2 of splicing factor arginine/serine-rich 7 [p/ 11.83]. Anp32a; Acidic leucine- rich nuclear phosphoprotein 32 [p/ 3.99]. 


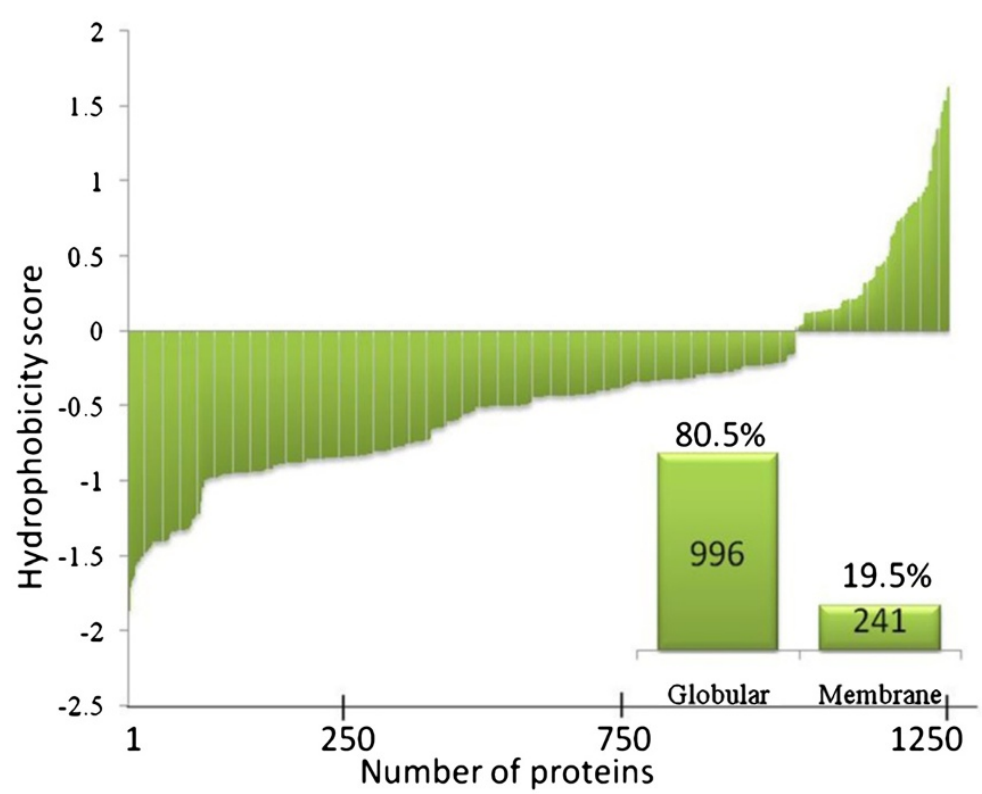

Figure 3 Gravy index score (average hydrophobicity and hydrophilicity) of colon proteins measured by Kyte-Doolittle and Hopp Woods formula. Hydrophobicity score (arbitrary unit) below 0 are more likely globular (hydrophilic protein), while scores above 0 are more likely membranous (hydrophobic). Inserted bar panel represents number and percentage of each group in colon proteome.

Result showed that out of 1237 proteins, 996 representing 80.5\% were globular (cytoplasmic) while 241 (19.5\%) were membranous (Figure 3). The relative small number of membrane-located proteins might be due to its hydrophobic resistance to be dissolved in the lysis buffer. Further optimization of hydrophobic proteins extraction is required.

\section{Subcellular localization of identified proteins}

We parsed protein dataset into subcellular localization using BiNGO. As shown in Figure 4, around 786 representing $64 \%$ of identified proteins were located in cytoplasm. This major compartment was mainly mitochondrial, cytoskeleton, and endoplasmic reticulum proteins (right bar panel). On the other hand, equal numbers of identified proteins were situated in nucleus and plasma membrane (17\%; 208 entries/each). A small percentage (2\%) of extracellular space proteins was also detected in our dataset.

\section{$\mathrm{GO}$ annotation and functional network analysis}

Enrichment and depletion analysis of mouse colon proteome were applied in the current experiment to speculate over and under- represented proteins. As illustrated in Figure 5, enrichment GO annotation showed successful retrieval of wide diversity of proteins (shown by node size and intense color) and reflecting a comprehensive, unbiased Lc-Ms/Ms approach. This observation is coincidental with non significant under representation of the most parent GO categories (colorless nodes in Figure 5B) with an exception of some daughter categories. For instance, membranous and nuclear proteins were shown to be under represented with $P$ value less than 0.01 (see also Additional file 3). Once again, this finding supports gravy index result and likelihood is due to the known difficulty to extract membranous proteins because of its hydrophobic nature. 


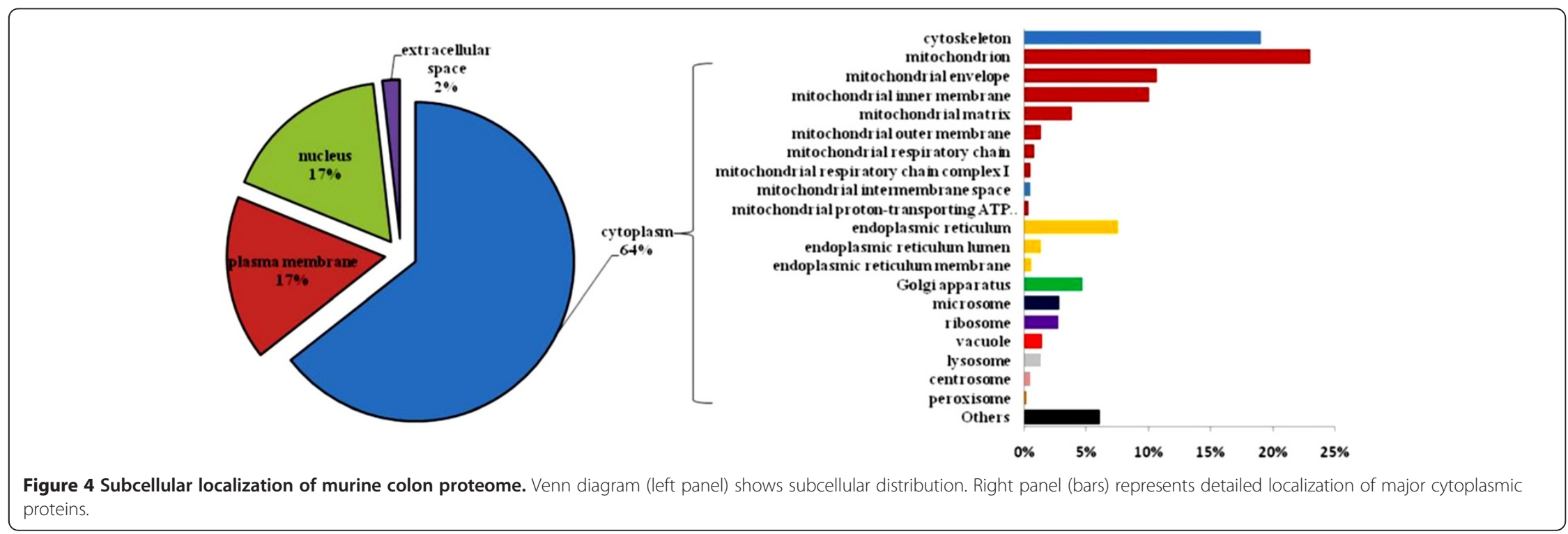




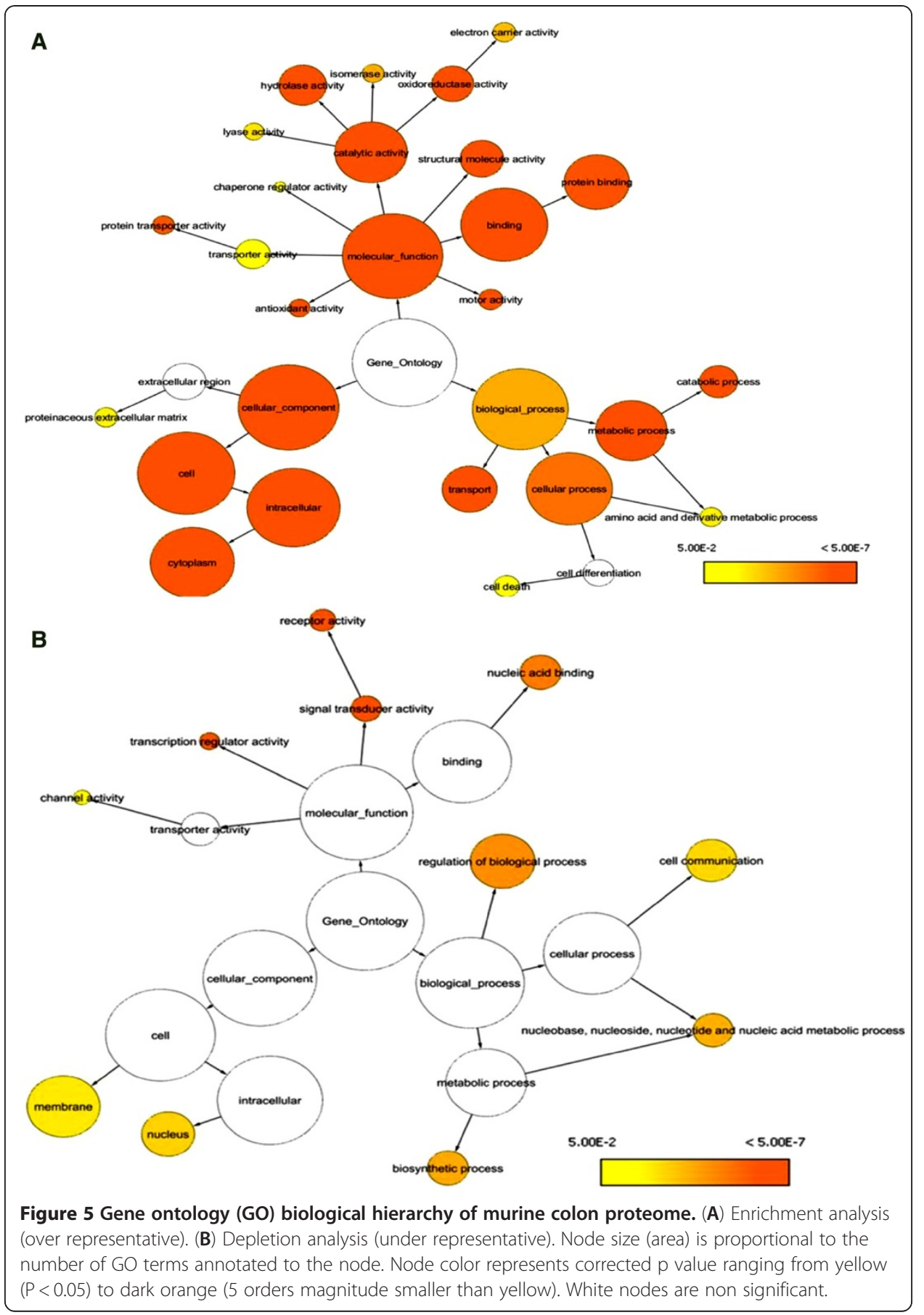

Functional analysis and protein family network illustrated in Figure 6 showed the involvement of 1199 identifiers in essential metabolic processes and pathways of the colon tissue (38 identifier were not recognized) and reflect the relationship between the terms based on similarity of their associated genes. As summarized in Figure 7, ten potential groups were recognized and defined with a leading term (to minimize the complex structure of GO tree) based on the highest significance scoring within the group. These enriched groups indicate their relevance in essential metabolic functions and 


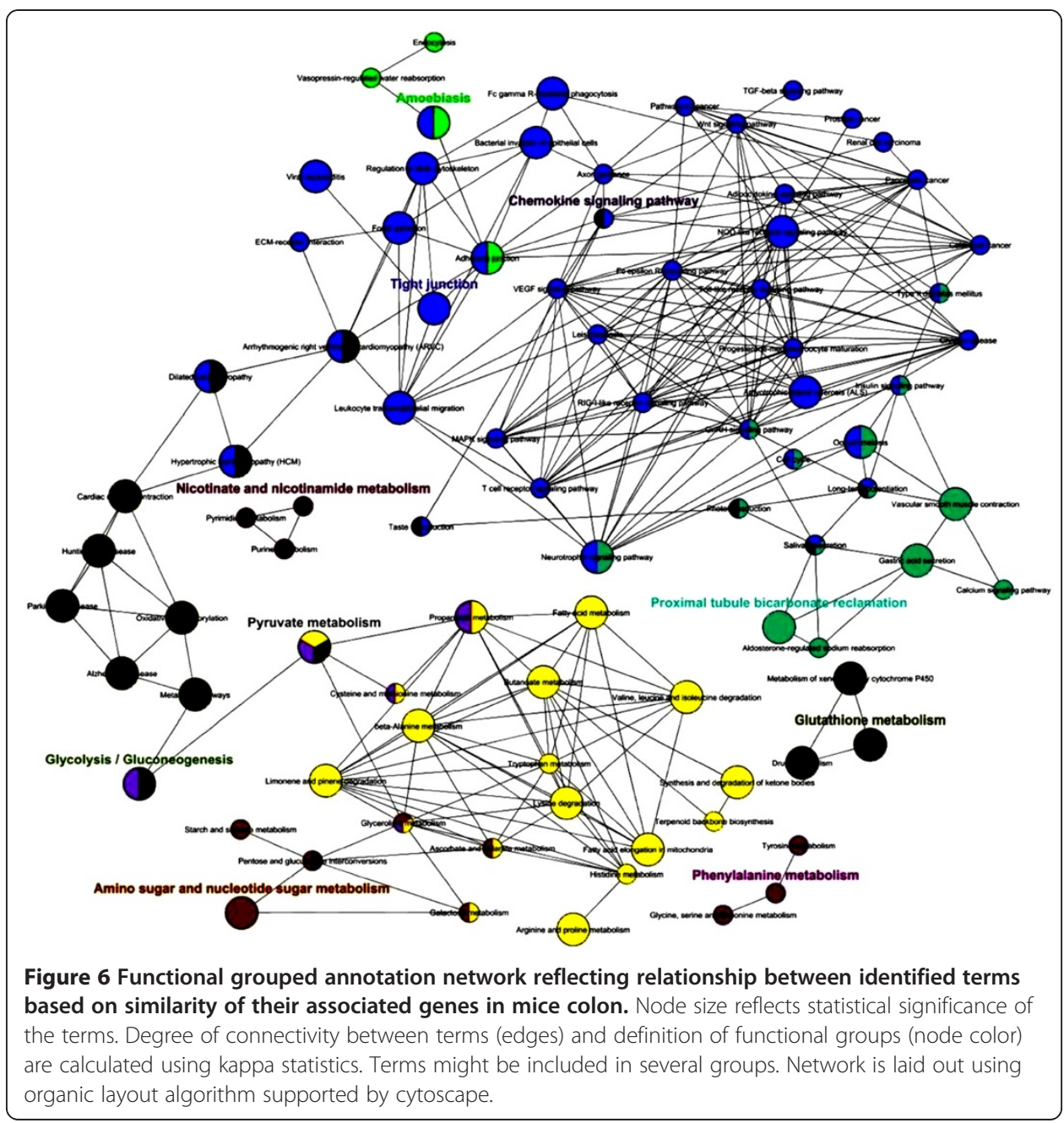

processes in colon tissue. Detailed sub functional term classification can be found in Additional file 1.

\section{Regulation of actin cytoskeleton/tight junction and related family groups}

Representing over one third (563) of identified terms, as illustrated in Figure 6, this family holds $43 \mathrm{GO}$ terms (shown by nodes). Most prominent is actin cytoskeleton which contains 47 identifiers. A wide variety of essential pathways could be recognized in this group. For example; MAPK (proliferation and apoptosis), VEGF (angiogenesis), Toll-like and T-like receptors (Immune barrier) signaling pathways and others, which reflect the active metabolic processes took place in the colonic cells. Moreover, several protein candidates for cancer pathways could be reported. Most notably Mapk8 and 9, Rac1 and 2, Rhoa for colorectal cancer, Cdc42, Fh1, Rac1, Rap1a, Tceb2 for renal cell carcinoma, Cdc42, Mapk8and 9, Pld1, Rac1and 2for pancreatic carcinoma and Fgfr2, Gstp1, Hsp90aa1, Hsp90ab1, Hsp90b1 for prostate cancer.

\section{Glycolysis and Glyconeogenesis and related family groups}

Major 2 ubiquitous processes that involve glucose breakdown (glycolysis) and its generation form non carbohydrate sources (glyconeogenesis) were detected. In colon proteome 


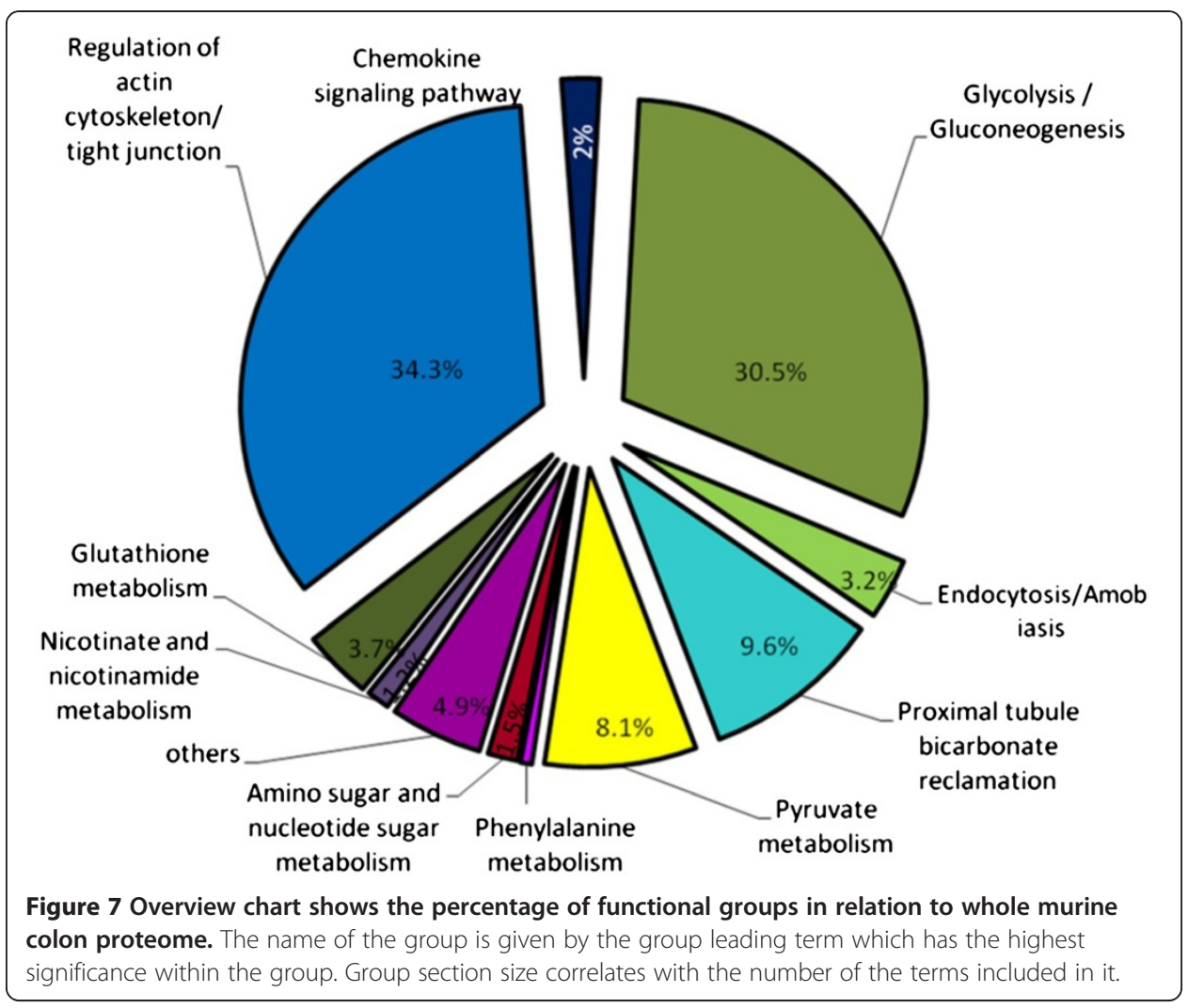

database, we reported 500 identifiers representing 30.5\% of total colon proteome representing Glycolysis pathway. This family holds wide members of enzymes including aldolases $A$ and $B$, alcohol dehydrogenases family members, enolases $(\alpha, \beta$, and $\gamma)$, lactate dehydrogenases, and others.

\section{Proximal tubule bicarbonate reclamation and related family groups}

This family includes ATPase, $\mathrm{Na}+\mathrm{K}+$ transporters [alpha1-4], glutamate dehydrogenase and malate dehydrogenase 1 (NAD) and representing 9.6\% of colon database. These catalytic enzymes are essential for exchanging sodium and potassium ions and providing energy for active transport of various nutrients in the gut. Other ATPase transporters were also identified which are contributors in salivary and gastric acid secretion such as (ATP1b1and ATP4a).

\section{Pyruvate metabolism and related family groups}

An essential group family which has key enzymes in citric acid cycle including dehydrogenases such as pyruvate, lactate, malate dehydrogenase. This group is mainly responsible for cellular respiration and release of energy via NADH. We were able also to recognize a wide variety of enzymes that contribute in amino acid metabolism and participating in cysteine, methionine, valine, leucine and isoleucine, arginine, proline, histidine and tryptophane synthesis and breakdown including LAP3, OAT, GOT2, and ALDH2 (Additional file 3). Several enzymes that share in fatty acid metabolism and elongation were also reported such as acetyl CoA acyltransferese 1 and 2, alcohol dehydrogenase 1 and 2 and others. 


\section{Glutathione metabolism and related family groups}

Includes glutathione peroxidase (GPX 1-5) and glutathione S- transferases (GST 1-5) enzymes that protect cells and other enzymes form oxidative damage by catalyzing the reduction of hydrogen peroxide, lipid peroxides and organic hydroperoxides.

\section{Other families}

Representing less than $15 \%$ of colon database and including chemokine signaling pathway, nicotinate and nicotinamide metabolism, amino sugar and nucleotide sugar metabolism, phenylalanine metabolism and amobiasis.

\section{Relative abundance of identified murine colon proteome}

In addition to protein identification and annotation, we determined also the relative protein abundance profiling of our murine colon proteome using 3 different approaches; NSAF, PAF, and emPAI owing to its extreme importance in comparative proteomics studies. These algorithms relay on the fact that spectral count (for NSAF and PAF) and peptide rank and score (for emPAI) correlate with relative protein abundance [17-19]. In Figure 8A, individual NSAF in the analyzed samples showed similar
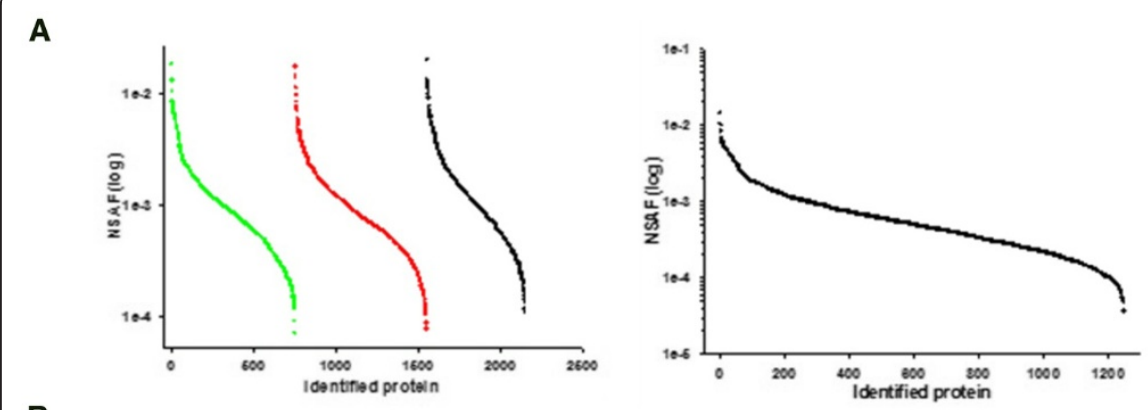

B
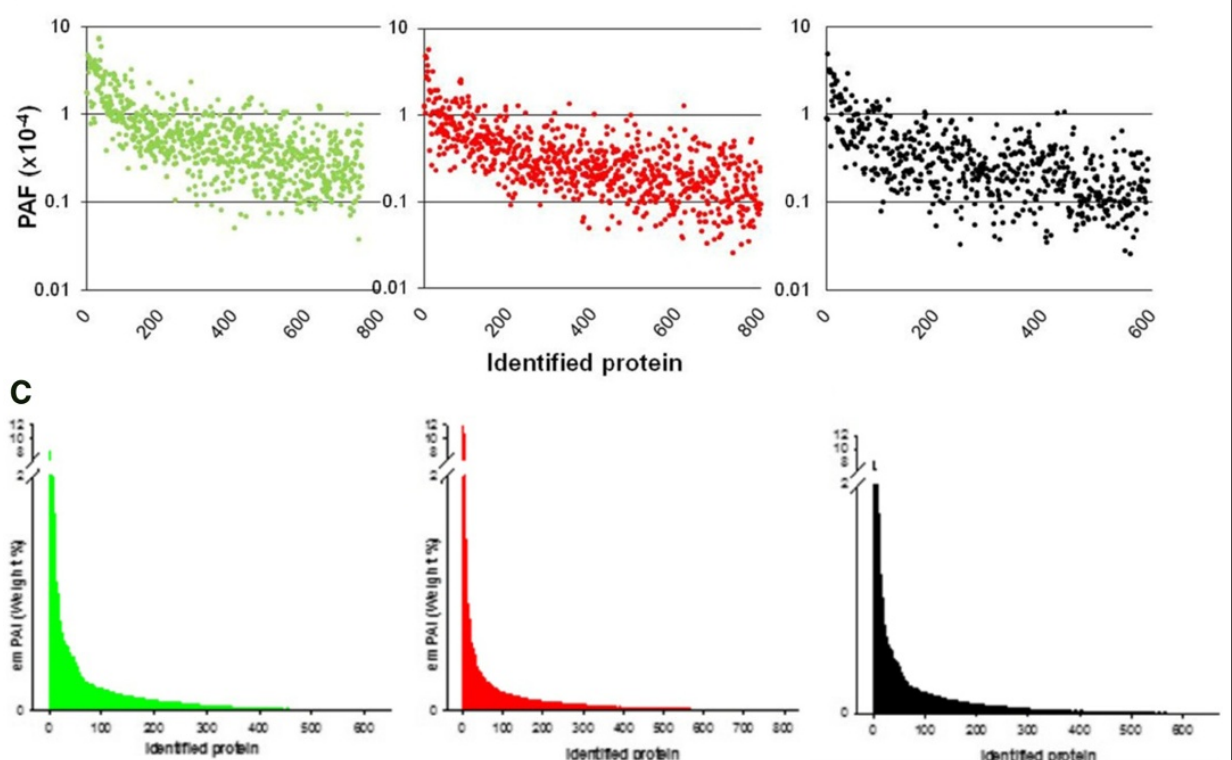

Figure 8 Relative quantification of murine colon proteins. (A) Normalized spectral abundance factor (NSAF) for each sample (left) and after merging (right). (B) Protein abundance factor (PAF). (C) Protein weight percentage calculated based on exponentially modified protein abundance factor (emPAl). 
pattern. Following construction of the colon proteome catalogue, protein abundance was fallen within the range from $1.2 \times 10^{-5}$ to $1.8 \times 10^{-2}$. This wide dynamic range further attests our unbiased Lc-Ms approach. When calculated using PAF algorithm, most of proteins were ranged from $0.1 \times 10^{-4}$ to $1.0 \times 10^{-4}$ (Figure $8 \mathrm{~B}$ ). We noticed the existence of several ubiquitous proteins in high abundance $(\sim 5$ folds $)$ such as transgelin, actin, and $ß$-globin which is recommended to be depleted when investigating low abundance proteins. On the other hand, several low abundance proteins were also identified. For instance, dipeptidyl - peptidase $1\left(0.03 \times 10^{-4}\right)$ and polymeric immunoglobulin receptor $\left(0.05 \times 10^{-4}\right)$. A similar pattern was also noticed when emPAI was used (Figure 8C). To compare between these 3 approaches, we sorted the top 30 abundant peptides (Additional file 4). Obviously, although several top abundant proteins were found to be shared between 3 algorithms, other candidates were different which probably might be due the fact that emPAI relies on peptide score while NSAF and PAF depends on spectral count.

\section{Comparison of murine colon proteome to gene expression database of mouse colon}

At last, we compared our generated murine colon proteome with the gene expression dataset for mouse colon in order to confirm the feasibility of this dataset as a standard reference for further colon experimentation. For that purpose, the whole length mouse colon genes were extracted from the reference expression dataset (RefEx) repository. The later was compared versus our generated proteome database (based on its gene ontology). As exemplarily shown in Figure 9, the current murine colon proteome showed an over lapping with around $35.6 \%$ compared to the known mouse colon genes. This result can be explained by the selective property and general limitation of mass spectrometry. Moreover, 13 genes were identified in our proteome dataset and have not been recognized in colon genome. These biased genes are possibly a non colon genes contamination.

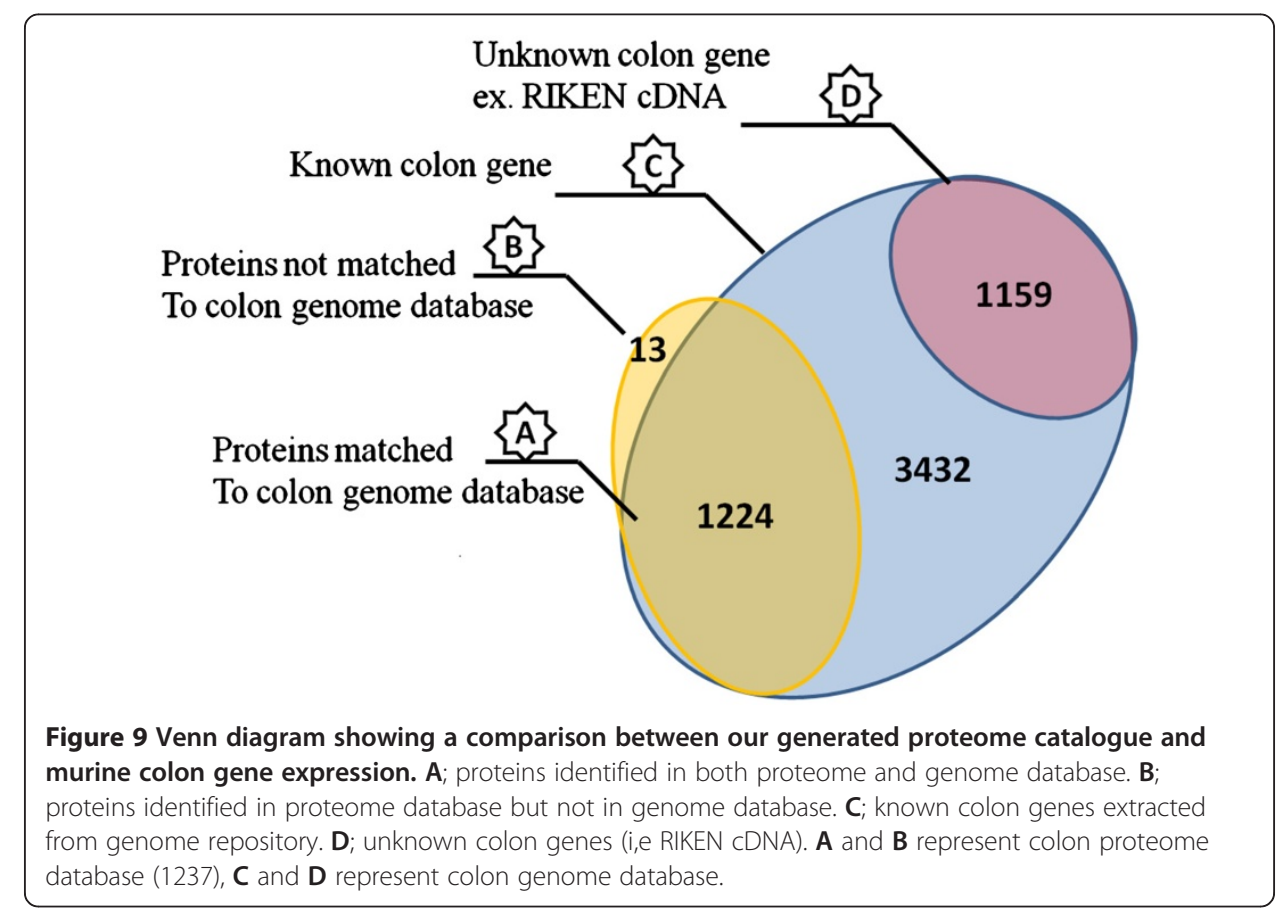




\section{Biological insights}

Recent advances in mass spectrometric methodologies enabled direct analysis of complex protein mixtures in a shotgun approach for global protein identification and biomarker discovery. Presented data in this article, provides not only a normal comprehensive colon proteome database, but also, various label free quantification methods for researcher's guidance especially when monitoring cancer- related colon protein expression. Furthermore, a functional network analysis of colon proteome is believed to provide a valuable piece of information for clarifying the relationship between possible predicted biomarkers. For instance, several candidates of gastrointestinal tract carcinoma showed correlated pattern; Mapk8 and Rac1,2 of colorectal cancer, cdc42 of renal cell carcinoma, pld1 in pancreatic cancer and Hsp90b1 and hsp90ab1 in prostate cancer. These data might anticipate in elucidating cell signaling and pathophysiological pathways.

\section{Conclusion}

The present research reports a comprehensive whole-tissue colon proteome catalogue consists of 1237 high confidence candidate protein with $\mathrm{FDR}<2$, together with its characteristics, relative expression, and functional pathway analysis which depicts unbiased database. Moreover, the colon protein profile shown in this report represents, to the best of our knowledge, a reference point for further comparative studies and better understanding protein expression patterns induced not only in normal physiological status but also in commonly diseased conditions as well.

\section{Additional files}

Additional file 1: Reproducibility and accuracy of Lc-Ms/Ms analysis for replicate samples.

Additional file 2: Murine colon proteome merged database.

Additional file 3: Murine colon biological hierarchy showing both enrichment and depletion analysis.

Additional file 4: NSAF \{bars representing sample replicate runs\}.

\section{Abbreviations}

DTT: Dithiothreitol; IAA: Iodoacetamide; LC-ESI-IT-TOF: Liquid chromatography- electospray ionization- time of flight; TFA: Trifluoruacetic acid; SDS-PAGE: Sodium dodecyl sulfate polyacrylamide gel electrophoresis; CID: MOWSE scoring, Molecular weight search scoring; GO: Gene ontology, colloidal ion dissociation; NSAF: Normalized spectral abundance factor; FDR: false discovary rate; PAF: Protein abundance factor; emPAl: exponentially modified protein abundance factor.

Competing interests

The authors have declared no conflict of interest.

Authors' contributions

SM: principally involved in experimental design, sample preparation, analysis and bioinformatic analysis, writing the article. YY carried out the mass spectrometric analysis. HL, YM and MY involved in animal care. SE,BX YZ and HF partially shared in protein alignment and network analysis. EY and SS carried out the genetic analysis and TY shared in experimental design and manuscript revision. All authors read and approved the final manuscript.

\section{Funding}

This work was supported by JSPS (Japan Society for Promotion of Science) Grant-in-Aid for scientific research (B) to SM (23790933) and Grant-in-Aid for scientific research (B) to TY (21390262) from Ministry of Education, Culture, Sports, Science and Technology of Japan. The funders had no role in study design, data collection and analysis, decision to publish, or preparation of the manuscript.

\section{Author details}

${ }^{1}$ Department of Structural Pathology, Institute of Nephrology, Graduate School of Medical and Dental Sciences, Niigata University, Niigata, Japan. ${ }^{2}$ Department of Physiology, Faculty of Veterinary Medicine, Suez Canal University, Suez Canal, Egypt. ${ }^{3}$ Department of Intensive Care Unit, West-China Hospital, Sichuan University, Sichuan, China. ${ }^{4}$ Animal resource 
branch, Center of Bio-based researches, Brain research Institute, Niigata University, Niigata, Japan. ${ }^{5}$ Department of Microbiology and Immunology, Faculty of Pharmacy, Suez Canal University, Suez Canal, Egypt. 'Department of Nephrology, Tokyo Medical and Dental University, Tokyo, Japan.

Received: 31 January 2012 Accepted: 24 July 2012

Published: 28 August 2012

\section{References}

1. Rose RC: Water-soluble vitamin absorption in intestine. Annu Rev Physiol 1980, 42:157-171.

2. Weiser MM, Bloor JH, Dasmahapatra A: Intestinal calcium absorption and vitamin D metabolism. J Clin Gastroenterol 1982, 4:75-86

3. Donini LM, Savina C, Cannella C: Nutrition in the elderly: role of fiber. Arch Gerontol Geriatr 2009, 49(Suppl 1):61-69.

4. Davis CD, Milner JA: Gastrointestinal microflora, food components and colon cancer prevention. J Nutr Biochem 2009, 20:743-752.

5. Gourley GR, Yang L, Higgins L, Riviere MA, David LL: Proteomic analysis of biopsied human colonic mucosa. J Pediatr Gastroenterol Nutr 2010, 51:46-54

6. Patel BB, Li XM, Dixon MP, Blagoi EL, Seeholzer SH, Chen Y, Miller CG, He YA, Tetruashvily M, Chaudhry AH, et al: Searchable high-resolution 2D gel proteome of the human colon crypt. J Proteome Res 2007, 6:2232-2238.

7. Lenaerts K, Bouwman FG, Lamers WH, Renes J, Mariman EC: Comparative proteomic analysis of cell lines and scrapings of the human intestinal epithelium. BMC genomics 2007, 8:91.

8. Yi H, Li XH, Yi B, Zheng J, Zhu G, Li C, Li MY, Zhang PF, Li JL, Chen ZC, Xiao ZQ: Identification of Rack1, EF-Tu and Rhodanese as aging-related proteins in human colonic epithelium by proteomic analysis. J Proteome Res 2010, 9:1416-1423.

9. Mazzanti R, Solazzo M, Fantappie O, Elfering S, Pantaleo P, Bechi P, Cianchi F, Ettl A, Giulivi C: Differential expression proteomics of human colon cancer. Am J Physiol 2006, 290:G1329-G1338.

10. Rho JH, Qin S, Wang JY, Roehrl MH: Proteomic expression analysis of surgical human colorectal cancer tissues: up-regulation of PSB7, PRDX1, and SRP9 and hypoxic adaptation in cancer. J Proteome Res 2008, 7:2959-2972.

11. Zhao L, Liu L, Wang S, Zhang YF, Yu L, Ding YQ: Differential proteomic analysis of human colorectal carcinoma cell lines metastasis-associated proteins. J Cancer Res Clin Oncol 2007.

12. Ramagli LS, Rodriguez LV: Quantitation of microgram amounts of protein in two-dimensional polyacrylamide gel electrophoresis sample buffer. Electrophoresis 1985, 6:559-563.

13. Magdeldin S, Li H, Yoshida Y, Satokata I, Maeda Y, Yokoyama M, Enany S, Zhang Y, Xu B, Fujinaka H, et al: Differential proteomic shotgun analysis elucidates involvement of water channel aquaporin 8 in presence of alpha-amylase in the colon. J Proteome Res 2010, 9:6635-6646.

14. Shevchenko A, Wilm M, Vorm O, Mann M: Mass spectrometric sequencing of proteins silver-stained polyacrylamide gels. Anal Chem 1996, 68:850-858.

15. Perkins DN, Pappin DJ, Creasy DM, Cottrell JS: Probability-based protein identification by searching sequence databases using mass spectrometry data. Electrophoresis 1999, 20:3551-3567.

16. Pappin DJ, Hojrup P, Bleasby AJ: Rapid identification of proteins by peptide-mass fingerprinting. Curr Biol 1993, 3:327-332.

17. Liu H, Sadygov R, Yates J: A model for random sampling and estimation of relative protein abundance in shotgun proteomics. Anal Chem 2004, 76:4193-4201.

18. Powell DW, Weaver CM, Jennings JL, McAfee KJ, He Y, Weil PA, Link AJ: Cluster analysis of mass spectrometry data reveals a novel component of SAGA. Mol Cell Biol 2004, 24:7249-7259.

19. Ishihama Y, Oda Y, Tabata T, Sato T, Nagasu T, Rappsilber J, Mann M: Exponentially modified protein abundance index (emPAl) for estimation of absolute protein amount in proteomics by the number of sequenced peptides per protein. Mol Cell Proteomics 2005, 4:1265-1272.

20. Maere S, Heymans K, Kuiper M: BiNGO: a Cytoscape plugin to assess overrepresentation of gene ontology categories in biological networks. Bioinformatics (Oxford, England) 2005, 21:3448-3449.

21. Bindea G, Mlecnik B, Hackl H, Charoentong P, Tosolini M, Kirilovsky A, Fridman WH, Pages F, Trajanoski Z, Galon J: ClueGO: a Cytoscape plug-in to decipher functionally grouped gene ontology and pathway annotation networks. Bioinformatics (Oxford, England) 2009, 25:1091-1093.

22. Shannon P, Markiel A, Ozier O, Baliga NS, Wang JT, Ramage D, Amin N, Schwikowski B, Ideker T: Cytoscape: a software environment for integrated models of biomolecular interaction networks. Genome Res 2003, 13:2498-2504

23. Hochberg Y, Benjamini Y: More powerful procedures for multiple significance testing. Stat Med 1990, 9:811-818

24. Ashburner M, Ball CA, Blake JA, Botstein D, Butler H, Cherry JM, Davis AP, Dolinski K, Dwight SS, Eppig JT, et al: Gene ontology: tool for the unification of biology. The Gene Ontology Consortium. Nat Genet 2000, 25:25-29.

25. Aoki KF, Kanehisa M: Using the KEGG database resource. Curr Protoc Bioinformatics 2005, Chapter 1:Unit 112.

26. Kyte J, Doolittle RF: A simple method for displaying the hydropathic character of a protein. J Mol Biol 1982, 157:105-132.

doi:10.1186/1756-0381-5-11

Cite this article as: Magdeldin et al: Murine colon proteome and characterization of the protein pathways. BioData Mining 2012 5:11. 